\title{
Silent information regulator 3: the Goldilocks of the silencing complex
}

\author{
Anne Norris ${ }^{1,2}$ and Jef D. Boeke ${ }^{1,2,3}$ \\ ${ }^{1}$ Department of Molecular Biology and Genetics, Johns Hopkins University School of Medicine, Baltimore, Maryland 21205, \\ USA; $^{2}$ The High Throughput Biology Center, Johns Hopkins University School of Medicine, Baltimore, Maryland 21205, USA
}

A recent explosion of work surrounds the interactions between Sir3p (Silent Information Regulator 3) and chromatin. We review here the Sir3p functions related to its role in silencing in Saccharomyces cerevisiae. This unusual protein, which is absolutely required for silencing, is distantly related to the highly conserved replication initiator Orc1p, but is itself phylogenetically limited to "postgenome-duplicated" budding yeasts. Several recent studies revise earlier models for Sir3p action. Specifically, the N-terminal bromo-adjacent homology (BAH) domain plays a now well-defined role in silencing, and a picture is emerging in which both termini of Sir3p bind two locations on the nucleosome: (1) the loss of ribosomal DNA silencing (LRS) surface in the nucleosome core, and (2) the N-terminal histone tails for effective silencing at telomeres. We relate Sir3p structure and function, and summarize recent molecular studies of Sir3p/chromatin binding, Sir3p/Dot1p competition, and the possible role of O-Acetyl ADP ribose (O-AADPR) in Sir3p/chromatin binding. We emphasize recent genetic data that provide important new insights and settle controversies created by in vitro work. Finally, we synthesize these ideas to revise the model for how Sir3p mediates silent chromatin formation in yeast, in part through its affinity for the LRS region of the nucleosome, which must be "just right."

Supplemental material is available at http://www.genesdev.org.

Classical silencing is typified by silent chromatin in Saccharomyces cerevisiae. Its heterochromatin-like structure occurs in three locus types: (1) silent matingtype cassettes $H M L$ and $H M R$, (2) telomeres, and (3) ribosomal DNA (rDNA) repeats (for review, see Rusche et al. 2003). All three share requirements for post-translational modification of the histone tails and core, as well as a Sir2p (Silent Information Regulator 2)/partner proteins "silencing complex." Most mechanistic data on silent chromatin formation come from studies on telomeric and HM locus silencing. The Sir complex at these loci consists of Sir1, Sir2, Sir3, and Sir4p, with Sirlp playing

[Keywords: SIR3; silencing; chromatin; telomere; SIR2; SIR4]

${ }^{3}$ Corresponding author.

E-MAIL jboeke@jhmi.edu; FAX (410) 502-1872.

Article is online at http://www.genesdev.org/cgi/doi/10.1101/gad.1865510. a significant role in the establishment of $H M$ locus silencing, variable roles at native telomeres, and only an indirect role at artificial truncated telomeres often used to study silencing. Sir2p is a histone deacetylase, while Sir1, Sir3, and Sir4p are thought to play more "structural" roles. It is interesting to note in this context that the Sir3p protein contains an AAA ATPase ("ATPases associated with a variety of cellular activities" domain) motif (see Fig. 1).

In the current model for telomeric silencing, Rap1 and $\mathrm{Ku}$ proteins bind directly to telomere chromatin, followed by recruitment of the Sir $2 / 3 / 4 p$ complex. Iterative cycles of Sir2p-mediated deacetylation of histone tail lysines, specifically at $\mathrm{H} 4 \mathrm{~K} 16$, create high-affinity sites for the Sir $2 / 3 / 4 \mathrm{p}$ complex in adjacent nucleosomes, allowing spreading of silent chromatin (Hoppe et al. 2002; Luo et al. 2002; Rusche et al. 2002; Tanny et al. 2004; Rudner et al. 2005). Encroachment of silent chromatin from silent loci into neighboring euchromatin is prevented by redundant competing mechanisms: antisilencers or "boundary elements," the inherent instability of the silencing complex antagonizing the silencingpromoting role of Sir $2 / 3 / 4 \mathrm{p}$, and other cis- and transacting factors (Lynch and Rusche 2009).

\section{Sir3p}

A most striking feature of Sir3p is its ability to condense chromatin even when the Sir $2 / 4$ complex is depleted, suggesting that some intrinsic properties of Sir3p allow it to bind to and condense nucleosomes (Hecht et al. 1996; Strahl-Bolsinger et al. 1997). Sir3p structure enables its role in silent chromatin. It has three distinct structural domains (Fig. 1): the N-terminal bromo-adjacent homology (BAH) domain, followed by a 300-residue stretch of intrinsically disordered amino acid residues, and then a C-terminal AAA ATPase that is highly conserved among diverse protein types (Callebaut et al. 1999; Duina and Winston 2004; Connelly et al. 2006; Hou et al. 2006; McBryant et al. 2006).

\section{BAH domain}

In Sir3p, the BAH domain consists minimally of amino acids 1-214 (see Figs. 1, 2; Callebaut et al. 1999; Connelly et al. 2006; Hou et al. 2006; Sampath et al. 2009). The 
A
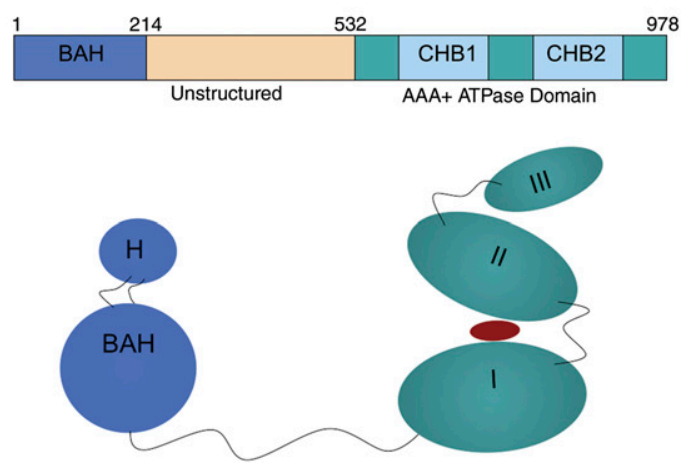

Figure 1. Domain structure of Sir3. (A) The primary domain structure of Sir3p with important domains highlighted in different colors. The first 214 amino acids comprise the BAH domain in blue; the region from amino acids 214 to $532(\tan )$ is largely unstructured (McBryant et al. 2006); and from amino acids 532 to 978 (green), Sir3p is similar to the CDC6 subfamily of the AAA ATPase domain. CHB1 and CHB2 (blue) indicate the $\mathrm{C}$-terminal histone-binding $(\mathrm{CHB})$ activity associated with this portion of Sir3p. The BAH domain also contains histone-binding activity. $(B)$ A cartoon version of the tertiary structure of Sir3 based on the crystal structures of the BAH domain 2FL7 (Hou et al. 2006) and the Archaeal cdc6 protein ortholog 1FNN (chain A) (Liu et al. 2000). The BAH domain is colored in blue, and the C-terminal domain is colored in teal. The red oval placed between domains I and II of the C-terminal region of Sir3 indicates a possible nucleotide moiety such as O-AADPR that could bind to Sir3p.

BAH domain's role in silencing has been underappreciated until recently for at least two reasons: Both recombinant protein production in Escherichia coli and N-terminal tags disrupt the N-terminal acetylation critical for both binding chromatin and Sir3p stability (Wang et al. 2004; Onishi et al. 2007; van Welsem et al. 2008). Only once Sir3p was expressed with a C-terminal tag was its autonomous ability to bind nucleosomes recognized.

The first clue that this domain regulated Sir3p function came from suppressor studies of the H4 K16Q nonmating phenotype. In the presence of sir3-D205N or sir3$W 86 R$, the H4 K16Q yeast strain regained mating ability (Johnson et al. 1990). Interestingly enough, this same BAH domain mutation, sir3-D205N, subsequently appeared in several screens, including suppression of a rap1-s allele (missing the Raplp C-terminal Sir3p interaction domain) and mutations in the nucleosome core (Thompson et al. 2003; Norris et al. 2008). Why would one mutation suppress so many loss-of-silencing mutations? As we suggest below, getting the Sir3p BAH domain to the nucleosome is not only the crux of Sir3pnucleosome interactions, but it is also necessary that this interaction is "just right." [An explanation of "Goldilocks" for nonnative English speakers. In the modern version of the fairy tale "Goldilocks and the Three Bears," mutated somewhat from the original by Southey (1834), the fair maiden Goldilocks visits the house of the bears and tries out their three bowls of porridge (hot, medium, and cool), their three chairs (large, medium, and small), etc., and concludes in each case that the one in the middle is "just right" for her.]

\section{C-terminal domain}

Long thought to be its "business end," the Sir3p C terminus dominates its known cellular roles (Fig. 1), including Rap1p, histone tail, and self-interactions (Johnson et al. 1990; Cockell et al. 1995; Hecht et al. 1995; Liu and Lustig 1996; Park et al. 1998; Luo et al. 2002). C-terminal residues 623-762 and 799-910 can bind H3 and H4 tails in vitro (Hecht et al. 1995). Sir3p's anti-parallel dimerization activity maps to residues 464-728 and 832-978 (King et al. 2006). The 464-728 region is also the minimal domain for Sir4 interaction (Chang et al. 2003; King et al. 2006). A crystal structure of the complex of an Archaeal ORC1 homolog with DNA demonstrates that this domain can bind ATP/ADP and DNA nonspecifically (Gaudier et al. 2007), suggesting that the C terminus of Sir3p may bind both DNA and proteins. Based on combined structure and sequence alignment data (Liu et al. 2000), the Sir3p C terminus can be divided into three domains typical of this family: Domain I consists of Sir3p residues $\sim 532-726$, domain II consists of residues from 727-831, and domain III consists of residues from 832978 (see Fig. 1; Liaw and Lustig 2006; A Norris and JD Boeke, unpubl.). In the $\mathrm{AAA}^{+}$family, the ATP-binding pocket lies between domains I and II, which contain several conserved motifs important for nucleotide binding. Sir3p is enigmatic because its $\mathrm{AAA}^{+}$domain lacks many residues important for $\mathrm{ADP} / \mathrm{ATP}$ binding, but secondary structure algorithms predict a similar overall topology to the $\mathrm{AAA}^{+}$family (Neuwald et al. 1999; Iyer et al. 2004; Gaudier et al. 2007). Domain III is the most variable among this class of proteins, and is probably important for auxiliary factor binding (Neuwald et al. 1999; Iyer et al. 2004; Gaudier et al. 2007). Because SIR3 is a duplicated gene, it is free to evolve faster and "neofunctionalize" (Ohno 1970) than a gene required for viability, like Orclp. The lack of conservation of the Sir3p C-terminal ATP-binding pocket could suggest that Sir3p binds a related nucleotide such as O-acetyl ADP ribose (O-AADPR) for activity (see below). Tethering studies employing Sir3-LexA fusions, recognizing binding sites adjacent to a telomeric $U R A 3$ reporter, have mapped the functional regions of Sir3p required post-recruitment to the last 144 amino acids (Lustig et al. 1996; Park et al. 1998; Liaw and Lustig 2006).

\section{Interaction with chromatin}

Initial in vitro experiments mapped the necessary and sufficient Sir3p-nucleosome interaction domains to the C-terminal residue blocks 623-762 and 799-910 of Sir3p and the histone tails of H3 and H4 (Hecht et al. 1995). Subsequent studies demonstrated that the nucleosome core was also important, as was the $\mathrm{N}$ terminus of Sir3p for nucleosome-Sir3p interaction. Surface plasmon resonance (SPR) experiments show that Sir3p can bind the $\mathrm{H} 3-\mathrm{H} 4$ tetramer in vitro, as can the BAH domain alone, 
A
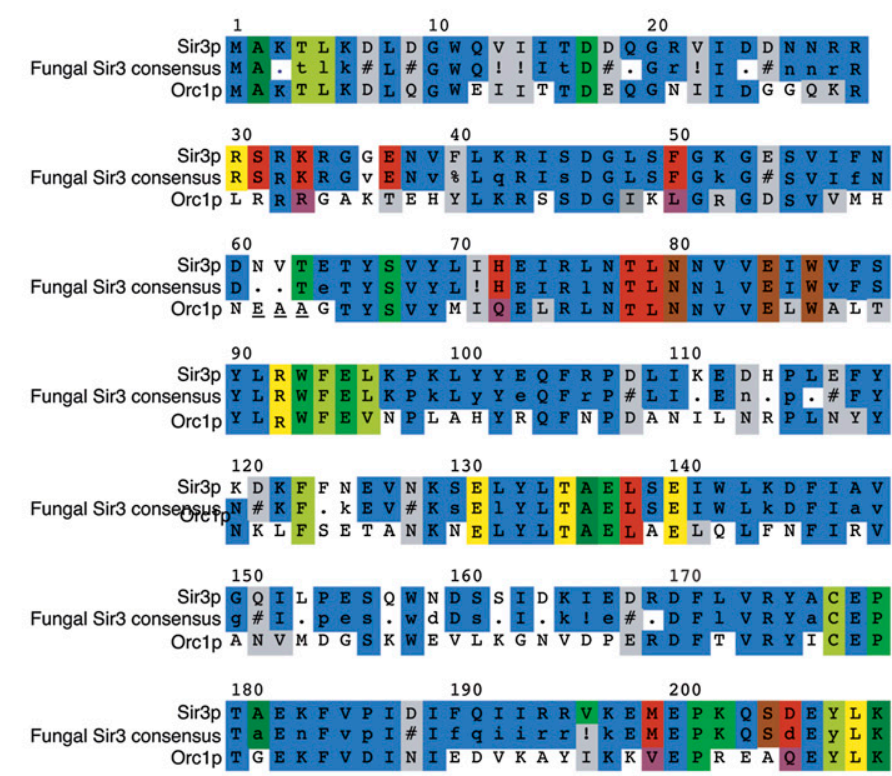

Orc1p T GE K F V D I N I E D V K A Y I K K V E P R E A Q E Y

210

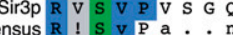

Orc1p D L T L

$\mathrm{V}, \$=\mathrm{LM}, \%=\mathrm{FY}, \#=\mathrm{BDN} \mathrm{DZ}, .=$ no consensus
KEY

$=$ Highly conserved residue
$=$ semiconserved residues

= LRS suppressors

"orc-like" and "semi orc-like" LRS suppressors

$=$ Dominant loss of silencing mutants

Loss of silencing mutatns

presence in more than one loss of function

screen

overlap between gain of function and loss

of function screen
B

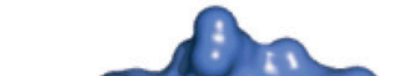

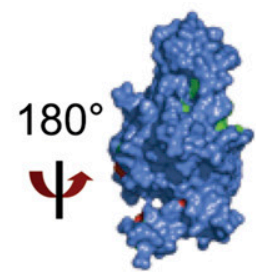

Figure 2. Critical binding surface of the BAH domain revealed by multiple genetic screens. (A) Multiple sequence alignment of SIR3 BAH domain from S. cerevisiae, consensus sequence for SIR3 BAH domain from post-genome duplication yeast strains, and BAH domain from ORC1. The side chains identified in the eso, DNG, and $1 \mathrm{fm}$ screens are highlighted in yellow-green, medium green, and light green, respectively; side chains from the slr screen are highlighted in red; residues overlapping between the slr screens and other loss-of-function screens are highlighted in brown; residues overlapping among the loss-of-function screens $D N G$, eso, and $1 \mathrm{fm}$ are highlighted in dark green. Mutations in the slr screen that introduce an "Orc-like" residue are highlighted in purple. Conserved residues are highlighted in blue, and semiconserved residues are highlighted in gray. (B) Surface representation of the crystal structure $2 \mathrm{FL} 7$ (Hou et al. 2006). The color coding is similar to $A$, but simplified; the $D N G$, eso, and $1 \mathrm{fm}$ screens are highlighted in green; the slr screen is highlighted in red; and the overlap between the two groups is highlighted in brown. The structure is rotated $180^{\circ}$ around the $X$-axis to demonstrate that all of the side chains identified by the various screens are found on one face of the BAH domain.

albeit with lower affinity, demonstrating that Sir3p can bind to nucleosomes independently of DNA /Onishi et al. 2007). Two recent elegant studies that reconstitute chromatin in vitro demonstrate the importance of both $\mathrm{H} 4$ K16 (tail) and H3 K79 (core) for Sir3 binding to nucleosome arrays (Johnson et al. 2009; Martino et al. 2009). Martino et al. (2009) purified Sir2, Sir3, and Sir4p from Sf9 cells and recombinant tailless Xenopus trinucleosome arrays and demonstrated that Sir3p could bind independently of histone tails, albeit with diminished affinity. The same study showed that Sir2,3,4p also bound to naked DNA, an activity attributed to Sir4 (Martino et al. 2009). Johnson et al. (2009) used Sir2, Sir3, and Sir4p from yeast extracts with recombinant nucleosomes and the HMR locus as a DNA template, creating a longer nucleosomal array. This study demonstrated that Sir3p can bind to nucleosome arrays without the Sir2/4 complex, but does so with much less affinity. Additionally, the Sir3p nucleosome interaction is disrupted by acetylation or mutation of $\mathrm{H} 4 \mathrm{~K} 16$, mutation of $\mathrm{H} 3 \mathrm{~K} 79$, or introduction of Htzl. All of these aberrations have much less effect on Sir3p binding to nucleosome arrays if Sir $2 / 4$ is added, at which point Sir2/3/4 binds irrespective of the status of H4 K16, while H3 K79A of Htzl only slightly reduces Sir3p nucleosome binding (Johnson et al. 2009). The interaction of Sir3p with modified nucleosomal arrays depends on the coiled-coil domain of Sir4 that binds Sir3, suggesting that Sir2/4 bridges Sir3 to modified chromatin (Johnson et al. 2009). Collectively, these results show that Sir3p binds both tail and nontail regions of the nucleosome in vitro, and that the Sir $2 / 4$ complex not only increases Sir3p's affinity for the nucleosome, but also makes it less sensitive to H4 K16 modification. This difference in affinity most likely reflects the initial recruitment and stable binding steps in silent chromatin assembly, and suggests that it may be Sir3p sensing the acetylation status of $\mathrm{H} 4 \mathrm{~K} 16$ and hence responsible for preventing further spread of the silencing complex. 
Importantly, studies in yeast cell extracts demonstrated that full-length Sir3p or ectopically expressed Sir3p BAH domain coimmunoprecipitate histone $\mathrm{H} 3$, and that this interaction is dependent on $\mathrm{H} 3$ residue 79 of the loss of rDNA silencing (LRS) surface and H4 tail residues 16, 17, and 19 . Sir3p prefers hypomethylated H3 K79 and hypoacetylated H4 K16 for its interaction with the nucleosome, as indicated by an increase in $\mathrm{H} 3$ coimmunoprecipitations (co-IPs) with Sir3p in $\operatorname{dot} 1 \Delta$ and sas2s cells (Onishi et al. 2007). These co-IP studies are corroborated by chromatin immunoprecipitation (ChIP) studies in which Sir3p does not chromatinimmunoprecipitate to telomeric DNA in an LRS mutant H3 A75V, or in tail mutants H4 K16Q or R17/19 A (Altaf et al. 2007; Fingerman et al. 2007; Norris et al. 2008). These studies suggest that the $\mathrm{H} 4$ tail and the LRS domain represent two surfaces important for Sir3p interaction with chromatin and, given that the $\mathrm{H} 4$ tail is flexible, they could represent a single contiguous surface stabilized in silent chromatin. Until recently, some controversy surrounded the exact region of Sir3p important for this interaction. Recombinant Sir3p C-terminal domain immunoprecipitates GST-tagged $\mathrm{H} 4$ peptides and an $\mathrm{H} 3$ peptide encompassing residues 68-83 (Hecht et al. 1995; Altaf et al. 2007). The interactions with the tail and the $\mathrm{H} 3$ residues surrounding $\mathrm{H} 3 \mathrm{~K} 79$ are modificationdependent, confirming the in vivo importance of the modification status of these two regions (Altaf et al. 2007). However, an ectopically expressed BAH domain can more efficiently coimmunoprecipitate $\mathrm{H} 3$ from a yeast wholecell extract than does full-length Sir3p, whereas Sir3p lacking the $\mathrm{BAH}$ domain fails to coimmunoprecipitate any $\mathrm{H} 3$, suggesting that the $\mathrm{C}$ terminus may inhibit the BAH domain to some extent (Onishi et al. 2007).

\section{Genetics seals the deal}

Whereas in vitro binding studies and whole-cell extract co-IPs can give a sense of the possible, genetics fills in the gaps in vivo. In the case of Sir3p, where in vitro interactions can be masked by requirements for nucleosome and Sir3p modification, recent genetic studies directly implicate the $\mathrm{BAH}$ domain as playing a critically important regulatory role in telomeric silencing. Four different screens isolated one surface of the SIR3 BAH domain as important for silencing in yeast in loss-offunction, dominant loss-of-function, and gain-of-function screens related to both telomeric and $H M$ locus silencing. A summary of these screens is represented in Figure 2 and Supplemental Table 1. For the sake of clarity in this publication, we classified the various screens as summarized below. The screen done by Stone et al. (2000) generated eso (for enhancer of sir one alleles). The screen done by Buchberger et al. (2008) produced DNG (dominantnegative alleles), the alleles isolated by Sampath et al. (2009) are named Ifm (for loss of function missense), and the alleles isolated by Norris et al. (2008) are named slr (for supressors of LRS).

The eso alleles enhance the mating defect of a $\operatorname{sir} 1 \Delta$ mutation. Normally, sir1s yeast mate at a very low but detectable level; the sir3 eso mutants can also mate, but abolish all mating in the sir1s strain (Stone et al. 2000). All mutations but one (S813A) localized to the BAH domain. The DNG screen necessarily isolated dominant loss of telomeric silencing SIR3 mutations, as it was performed in a $S I R 3^{+}$background. The screen isolated mutants that were somewhat defective in binding to telomeric chromatin but much more defective in spreading (Buchberger et al. 2008). This screen isolated many mutations clustered on the BAH domain and, as in the eso screen, isolated a single distinct mutation in the C-terminal region (L738P) that was the weakest in terms of its effects on telomeric silencing. While these screens revealed that the $\mathrm{BAH}$ domain is crucial for silencing, the slr screen as well as mutagenesis by Sampath et al. (2009) revealed a genetic link between the BAH domain and the LRS region of the nucleosome. In the slr screen, SIR3 alleles D205N and L79I, along with histone alleles H3 D77N and H4 H75Y, were isolated as LRS suppressors. The same histone alleles as well as SIR3 D205N were identified as suppressors of the sir3 A2T loss-of-silencing mutation (Norris et al. 2008; Sampath et al. 2009). Additionally, the subset of the $1 \mathrm{fm}$ and $D N G$ alleles failed to coimmunoprecipitate histone $\mathrm{H} 3$ from whole-cell extract or to chromatin-immunoprecipitate telomeric DNA, demonstrating that BAH is crucial for chromatin interaction. It is interesting that these loss-of-function alleles were dominant; presumably, the dominance reflects essential Sir3p multimerization, with loss of function leading to a poisoned subunit. The slr screen differed in that it isolated alleles of the BAH domain that suppressed histone LRS alleles' loss of telomeric silencing. Indeed, these mutations suppressed multiple LRS histone alleles, but not other nucleosome mutations that interfered with telomeric silencing. Informatively, the slr alleles resulted in paradoxically decreased telomeric silencing in the presence of wild-type histones, despite a significant increase in ChIP signal to telomeric DNA compared with wild-type Sir3p (Norris et al. 2008). Similarly, H3 K56Q mutations disrupt telomeric silencing but do not decrease the ability of the SIRs to chromatin-immunoprecipitate to telomeric DNA (Xu et al. 2007). These results suggest that SIR binding is a prerequisite for silencing but is not sufficient. The question remains: Why would "wild-type" histones lose silencing in the presence of SIR3 suppressor alleles D205N and L79I, but still chromatin-immunoprecipitate ample Sir3p to telomeric DNA? This seeming conundrum was recapitulated by studies with Orclp-Sir3p hybrids. Whereas an ectopically expressed Orclp BAH domain coimmunoprecipitates more histone $\mathrm{H} 3$ than Sir3p BAH, Orc1p BAH does not substitute well for Sir3p BAH in silencing (Stone et al. 2000; Onishi et al. 2007). Hence, we propose that Sir3p follows the "Goldilocks principle" (Southey 1834): Just as in the case of the Orclp BAH domain, when Sir3p binds too strongly to chromatin, it is counterproductive for telomeric silencing (Norris et al. 2008). Sir3p may need to either bind and release telomeric DNA for proper silencing or, alternatively, bind in more than one conformation, one of which involves 
the interaction of both BAH and LRS surfaces. Following the presumed gene duplication event that gave rise to SIR3, and during its "neofunctionalization" (Ohno 1970), Sir3p's BAH may have evolved a lower affinity for the LRS surface, consistent with the fact that Sir3p seems to have at least one additional distinct chromatin-binding domain (Hecht et al. 1995; Altaf et al. 2007; Onishi et al. 2007; Buchberger et al. 2008). Both the DNG and eso screens isolated single mutations in the $\mathrm{C}$ terminus of Sir3p, L738P, and S813A, respectively. Using GST-tagged $\mathrm{H} 4$ peptides, the L738P mutation interacted with $\mathrm{H} 4$ peptides more strongly than wild type, but, unlike all of the other DNG mutations, L738P was not defective in co-IP of $\mathrm{H} 3$, suggesting strong binding of the $\mathrm{C}$ terminus to $\mathrm{H} 4$ tails could also be deleterious to silencing (Buchberger et al. 2008). A picture emerges in which a balance needs to be struck between Sir3p BAH and C-terminal domains for binding to the nucleosome; this balance is crucial for effective silent chromatin formation.

\section{Electrostatic repulsion dominates the interaction}

The slr screen shows an electrostatic trend in the Sir3p BAH suppressors isolated, in that they tend to increase the positive charge of the BAH domain. Additionally, the $D N G$ screen also isolated two positively charged lysines on the BAH surface that lead to loss of silencing. This trend is strikingly similar to a complementary electrostatic trend on the LRS surface of the nucleosome, in which mutations that increase positive charge tend to increase telomeric silencing, whereas mutations that decrease positive charge tend to lose silencing. The simplest explanation for these data is that the Sir3p BAH domain makes direct contact with the LRS nucleosomal domain, and electrostatic repulsion dominates this interaction. The nucleosome and Sir3p normally repel each other in wild-type yeast to a certain extent, and it is the slightly unstable nature of this interaction that determines the strength of silencing (Norris et al. 2008). Thus, these slr mutants decrease potential repulsion, presumably by increasing nucleosome-binding affinity. In contrast, charge-neutralizing substitutions in the two lysines K202 and K209 on $\alpha$ helix 8 of Sir3p that, by this electrostatic hypothesis, should make positive contributions to Sir3p nucleosome binding were isolated in both the DNG and $1 \mathrm{fm}$ screens (Buchberger et al. 2008; Sampath et al. 2009). Furthermore, mutations on the LRS surface of the nucleosome that decrease the charge repulsion of D77 also suppress the loss of silencing of the SIR3 A2T or the LRS allele H3 A75V. Therefore, the crux of the interaction between Sir3p and the nucleosome is getting the BAH domain of Sir3p to the LRS surface of the nucleosome.

The collective interpretation of the phenotypes of these mutants is that (1) there is a functional surface on the BAH domain that interacts with the LRS surface of the nucleosome, and (2) the D205 residue makes an important repulsive contribution that limits nucleosomebinding affinity. In situations where Sir3p is able to bind all over the genome, as occurs in $\operatorname{dot} 1 \Delta$ or sas $2 \Delta$ cells, telomere silencing is lost; hence, it is important to (1) get Sir3p to the right place and (2) balance its affinity for the nucleosome. Like Goldilocks, the Sir3p BAH domain needs its affinity for the LRS surface of the nucleosome to be "just right."

Furthermore, weakening BAH-binding affinity might facilitate a role requiring alternating binding and release of chromatin or allowing for conformational changes that might be required for silencing. The simplest explanation is that Sir3p requires a distinct "sweet spot" in between a stable LRS-BAH interaction and one that is sufficiently weak to allow the necessary dynamic structural changes.

\section{Competition with Dot1p}

Dotlp methylation in euchromatin helps keep silent chromatin from spreading and the Sir $2,3,4 \mathrm{p}$ complex properly localized (van Leeuwen and Gottschling 2002; van Leeuwen et al. 2002). By ChIP analysis, both deletion and overexpression of DOT1 led to mislocalization of the Sir2,3,4p complex. Sir3p coimmunoprecipitates more H3 in both dot1 deletion and H4K16R strains in a Sir3p BAH domain-dependent manner (Onishi et al. 2007). However, the Sir3p C terminus inhibits Dotlp in vitro methylation reactions of $\mathrm{H} 3$ (Altaf et al. 2007; Fingerman et al. 2007), possibly by competing for the Dotlp-binding site. While Dot 1p most certainly binds the LRS region, residues 17 , 18 , and 19 of the H4 tail are also important for the methylation reaction (Fingerman et al. 2007). The C terminus of Sir3p was known to interact in vitro with the $\mathrm{H} 4$ tail, and hence could be competing with that region for binding (Hecht et al. 1995). Considering that the $\mathrm{H} 4$ tail emerges from a nearby nucleosome surface and is flexible and short, it is entirely possible that it can form a contiguous surface with the LRS domain for binding of Sir3p, Dotlp, or both. A related study found that the Sir3p C terminus could bind a peptide consisting of residues $68-83$ of $\mathrm{H} 3$, and this binding was methylationsensitive (Altaf et al. 2007). It appears that both the BAH C-terminal domains have the potential to bind the LRS region and the $\mathrm{H} 4$ tail, and Dotlp competes with both domains of Sir3p for this surface. While Dotlp is insensitive to H3 K79 methylation status, Sir3p binds only to the unmethylated surface; this competition between Sir3p and Dot $1 p$ helps dictate whether chromatin is silenced or expressed.

\section{O-AADPR}

One very tantalizing idea is that Sir3p does not bind ADP or ATP, but has evolved to bind the related molecule O-AADPR, a specific product of the $\mathrm{NAD}^{+}$-dependent Sir2p-mediated deacetylation reaction (Tanner et al. 2000; Sauve et al. 2001). There is striking evidence both for and against this idea. When O-AADPR is added to purified Sir2, Sir3, Sir4, and nucleosomes, filaments visible in the electron microscope (EM) are formed (Liou et al. 2005; Onishi et al. 2007). The most striking evidence for O-AADPR binding is that Sir2,3,4 complexes or Sir3p alone purified from baculovirus-infected cell 
extract have an increased affinity for trinucleosomes, as measured by gel shift when O-AADPR is added but not when analogs such as AADPR, ADP, ATP, or nonhydrolyzable ATP analogs are added (Martino et al. 2009). The effect was dramatic with the Sir2,3,4p complex, but is also quite significant with Sir3p alone, suggesting that it is the key target (Martino et al. 2009). The evidence against this hypothesis is based on a Sir3p-Hos3 fusion protein used to demonstrate that telomeric silencing can occur in the absence of O-AADPR (Chou et al. 2008). Hos3 is an $\mathrm{NAD}^{+}$-independent deacetylase that does not produce O-AADPR, nor does it normally deacetylate histones. When fused in-frame to the $\mathrm{C}$ terminus of Sir3p, it deacetylates histones at silent loci as measured by ChIP, and this effectively silences DNA /Chou et al. 2008). However, while it does not silence as well as wildtype yeast, it does silence as well as a Sir3-Sir2p fusion protein (Chou et al. 2008). Taken together, these experiments suggest that O-AADPR may indeed increase the affinity of Sir3p in the Sir2,3,4p complex for chromatin and increase silencing, but the small molecule is not absolutely required for silencing. It would be interesting to know whether O-AADPR is required for the type of silencing that occurs when Sir3p is overexpressed, leading to regions of silent DNA depleted of Sir2p and Sir4p, but enriched for Sir3p. Additionally, a crystal structure of the C terminus would shed light on whether Sir3p really does have a similar structure as the $\mathrm{AAA}^{+}$family, as has been predicted sequence alignments, and whether it can bind a nucleotide.

\section{Model for Sir3p and telomeric silent chromatin formation}

Silencing is a dynamic feature of telomeric DNA; there are silencing-promoting activities in the cell that are balanced with destabilizing activities that ultimately separate the silent regions from the expressed regions of the genome. The Sir3p BAH and C-terminal regions play critical roles in this balance (see Fig. 3). Establishment of silencing occurs through Raplp and the Ku complex binding to nonnucleosomal telomeric repeats at the chromosome tips. Raplp then recruits Sir3p and the Sir $2 / 4 p$ complex. The deacetylation activity of Sir $2 p$ then creates a high-affinity site for the SIR complex, allowing the SIR complex and silencing to spread. Several actions inhibit silencing: One is the inherent instability of the SIR complex, and the second is the competition between Sir2p and Sas2p for the acetylation state of histone H4 K16. The inherent instability can be exacerbated or counteracted by poorly defined cis-acting sequences, presumably Rap1-, Abf1-, and/or Orc1-binding sites; ARS sequences; and other sequences in the $\mathrm{X}$ core and $\mathrm{Y}^{\prime}$ domains of the telomeres (Boscheron et al. 1996; Fourel et al. 1999, 2002; Pryde and Louis 1999; Cheng and Gartenberg 2000; Mondoux and Zakian 2007). Acetylation of H4 K16 leads to deposition of histone variant HTZ1 and decreased affinity of the SIR complex for the nucleosomes, and especially for that of Sir3p and the nucleosome. This loss of affinity is reinforced by the

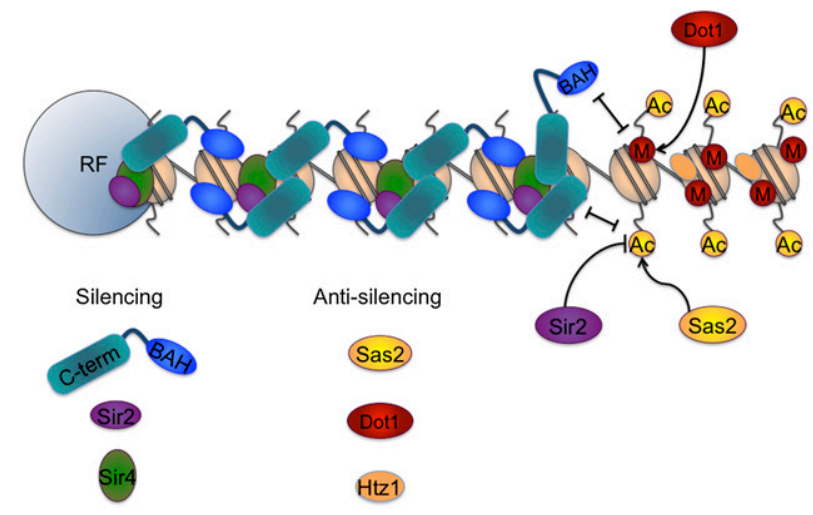

Figure 3. Competing actions for silencing followed by reinforcements. This figure is largely based on Altaf et al. (2007) and Buchberger et al. (2008). There are silencing-promoting actions such as Sir2p deacetylating the histone tails, which creates a high-affinity site for the SIR complex. Several actions inhibit silencing: One is the inherent instability of the SIR complex, and a second is the competition between Sir2p and Sas2p for the acetylation state of histone H4 K16. HTZ1 deposition also contributes to the destabilization of silencing (Venkatasubrahmanyam et al. 2007). Acetylation of H4 K16 leads to decreased affinity of the SIR complex for the nucleosomes and especially for that of Sir3 and the nucleosome; this loss of affinity is reinforced by the competition between Dotlp and Sir3p for the methylation status of histone H3 K79. Sir3p binds the LRS surface of the nucleosome, which encompasses H3 K79 and occludes its methylation by Dot1p; conversely, when H3 K79 is methylated, Sir3p binding is blocked. The orientation of Sir3p is meant to suggest that the $\mathrm{N}$-terminal $\mathrm{BAH}$ and the $\mathrm{C}$ terminus of Sir3p may bind to both the LRS domain and the histone $\mathrm{N}$-terminal tails, and that this dual binding may in fact be crucial to compaction of chromatin and silencing. Additionally, the Sir3 $\mathrm{C}$ terminus is shown to be most sensitive to the actelyation of H4 K16, which inhibits Sir3 "lockdown" onto the chromatin (Johnson et al. 2009), and hence spreading of the silent chromatin. Sir4p is depicted as binding to linker DNA as suggested in Martino et al. (2009), but this aspect of the model should be considered preliminary. The stoichiometry of the SIR complex is also preliminary, and is based primarily on Kimura and Horikoshi (2004) and Johnson et al. (2009).

competition between Dot1p and Sir3p for the methylation status of histone $\mathrm{H} 3 \mathrm{~K} 79$, as well as forces that act genome-wide such as HTZ1 deposition and H3 K4 methylation (Venkatasubrahmanyam et al. 2007). Mutations such as H3 K79R may disrupt some of the Dotlp anti-silencing activity, but all of the other anti-silencing mechanisms are still in place, leading to only a minor defect in silencing. The Sir3p BAH domain binds the LRS surface of the nucleosome that encompasses H3 K79 and occludes its methylation by Dotlp; conversely, when H3 K79 is methylated, Sir3p binding is blocked. The C-terminal region may also play a secondary role in binding the LRS surface, but seems to be most important for the establishment of silencing. The $\mathrm{C}$ terminus has several important roles, especially in the initiation of silencing: It binds Raplp for recruitment to the telomeric DNA; it recognizes the modification status of $\mathrm{H} 4 \mathrm{~K} 16$; it binds Sir4p, which intensifies its affinity for chromatin; 
and, importantly for spreading, it contains the antiparallel dimerization domain. If these prerequisites are not met, silencing is impaired. However, the crux of getting the right balance of silencing in the cell is getting the Sir3p BAH domain to the LRS surface of the nucleosome for which the affinity must be "just right": Too tight and silencing is lost; too loose and silencing is also lost. Just how "too tight" is counterproductive for silencing in molecular terms remains to be determined. Two models have been put forth here: Sir3p may need to bind in more than one conformation or, alternatively, Sir3p may need to bind and release for proper chromatin formation. We favor the model where Sir3p binds in more than one conformation; this is based largely on the fact that Sir3p has multiple nucleosome interaction domains as well as its own dimerization domain. Additionally, Sir3p binds with a different affinity and stoichiometry, depending on whether it is binding a mono-, tri-, or multinucleosomal array. It will be interesting to find out more clearly how Sir3p mediates compaction of silent chromatin and what, if any, role a small molecule such as O-AADPR could play in that process.

\section{Acknowledgments}

We thank the Boeke laboratory for helpful discussions, and reviewers of this paper for pointing out important recent information. Work on silencing was supported by NIH Roadmap grant U54 RR020839 to J.D.B.

\section{References}

Altaf M, Utley RT, Lacoste N, Tan S, Briggs SD, Cote J. 2007. Interplay of chromatin modifiers on a short basic patch of histone $\mathrm{H} 4$ tail defines the boundary of telomeric heterochromatin. Mol Cell 28: 1002-1014.

Boscheron C, Maillet L, Marcand S, Tsai-Pflugfelder M, Gasser SM, Gilson E. 1996. Cooperation at a distance between silencers and proto-silencers at the yeast HML locus. EMBO J 15: 2184-2195.

Buchberger JR, Onishi M, Li G, Seebacher J, Rudner AD, Gygi SP, Moazed D. 2008. Sir3-nucleosome interactions in spreading of silent chromatin in Saccharomyces cerevisiae. Mol Cell Biol 28: 6903-6918.

Callebaut I, Courvalin JC, Mornon JP. 1999. The BAH (bromoadjacent homology) domain: A link between DNA methylation, replication and transcriptional regulation. FEBS Lett 446: 189-193.

Chang JF, Hall BE, Tanny JC, Moazed D, Filman D, Ellenberger T. 2003. Structure of the coiled-coil dimerization motif of Sir4 and its interaction with Sir3. Structure 11: 637-649.

Cheng TH, Gartenberg MR. 2000. Yeast heterochromatin is a dynamic structure that requires silencers continuously. Genes \& Dev 14: 452-463.

Chou CC, Li YC, Gartenberg MR. 2008. Bypassing Sir2 and O-acetyl-ADP-ribose in transcriptional silencing. Mol Cell 31: 650-659.

Cockell M, Palladino F, Laroche T, Kyrion G, Liu C, Lustig AJ, Gasser SM. 1995. The carboxy termini of Sir4 and Rap1 affect Sir3 localization: Evidence for a multicomponent complex required for yeast telomeric silencing. J Cell Biol 129: 909-924.

Connelly JJ, Yuan P, Hsu HC, Li Z, Xu RM, Sternglanz R. 2006. Structure and function of the Saccharomyces cerevisiae Sir3 BAH domain. Mol Cell Biol 26: 3256-3265.
Duina AA, Winston F. 2004. Analysis of a mutant histone H3 that perturbs the association of Swi/Snf with chromatin. Mol Cell Biol 24: 561-572.

Fingerman IM, Li HC, Briggs SD. 2007. A charge-based interaction between histone $\mathrm{H} 4$ and Dot 1 is required for H3K79 methylation and telomere silencing: Identification of a new trans-histone pathway. Genes \& Dev 21: 2018-2029.

Fourel G, Revardel E, Koering CE, Gilson E. 1999. Cohabitation of insulators and silencing elements in yeast subtelomeric regions. $E M B O$ J 18: 2522-2537.

Fourel G, Lebrun E, Gilson E. 2002. Protosilencers as building blocks for heterochromatin. Bioessays 24: 828-835.

Gaudier M, Schuwirth BS, Westcott SL, Wigley DB. 2007. Structural basis of DNA replication origin recognition by an ORC protein. Science 317: 1213-1216.

Hecht A, Laroche T, Strahl-Bolsinger S, Gasser SM, Grunstein M. 1995. Histone H3 and H4 N-termini interact with SIR3 and SIR4 proteins: A molecular model for the formation of heterochromatin in yeast. Cell 80: 583-592.

Hecht A, Strahl-Bolsinger S, Grunstein M. 1996. Spreading of transcriptional repressor SIR3 from telomeric heterochromatin. Nature 383: 92-96.

Hoppe GJ, Tanny JC, Rudner AD, Gerber SA, Danaie S, Gygi SP, Moazed D. 2002. Steps in assembly of silent chromatin in yeast: Sir3-independent binding of a Sir2/Sir4 complex to silencers and role for Sir2-dependent deacetylation. Mol Cell Biol 22: 4167-4180.

Hou Z, Danzer JR, Fox CA, Keck JL. 2006. Structure of the Sir3 protein bromo adjacent homology (BAH) domain from $S$. cerevisiae at 1.95 A resolution. Protein Sci 15: 1182-1186.

Iyer LM, Leipe DD, Koonin EV, Aravind L. 2004. Evolutionary history and higher order classification of $\mathrm{AAA}^{+}$ATPases. I Struct Biol 146: 11-31.

Johnson LM, Kayne PS, Kahn ES, Grunstein M. 1990. Genetic evidence for an interaction between SIR3 and histone $\mathrm{H} 4$ in the repression of the silent mating loci in Saccharomyces cerevisiae. Proc Natl Acad Sci 87: 6286-6290.

Johnson A, Li G, Sikorski TW, Buratowski S, Woodcock CL, Moazed D. 2009. Reconstitution of heterochromatin-dependent transcriptional gene silencing. Mol Cell 35: 769-781.

Kimura A, Horikoshi M. 2004. Partition of distinct chromosomal regions: Negotiable border and fixed border. Genes Cells 9: 499-508.

King DA, Hall BE, Iwamoto MA, Win KZ, Chang JF, Ellenberger T. 2006. Domain structure and protein interactions of the silent information regulator Sir3 revealed by screening a nested deletion library of protein fragments. I Biol Chem 281: 20107-20119.

Liaw H, Lustig AJ. 2006. Sir3 C-terminal domain involvement in the initiation and spreading of heterochromatin. Mol Cell Biol 26: 7616-7631.

Liou GG, Tanny JC, Kruger RG, Walz T, Moazed D. 2005. Assembly of the SIR complex and its regulation by O-acetylADP-ribose, a product of NAD-dependent histone deacetylation. Cell 121: 515-527.

Liu C, Lustig AJ. 1996. Genetic analysis of Raplp/Sir3p interactions in telomeric and HML silencing in Saccharomyces cerevisiae. Genetics 143: 81-93.

Liu J, Smith CL, DeRyckere D, DeAngelis K, Martin GS, Berger JM. 2000. Structure and function of Cdc6/Cdc18: Implications for origin recognition and checkpoint control. Mol Cell 6: 637-648.

Luo K, Vega-Palas MA, Grunstein M. 2002. Rap1-Sir4 binding independent of other sir, $\mathrm{yKu}$, or histone interactions initiates the assembly of telomeric heterochromatin in yeast. Genes \& Dev 16: 1528-1539. 
Lustig AJ, Liu C, Zhang C, Hanish JP. 1996. Tethered Sir3p nucleates silencing at telomeres and internal loci in Saccharomyces cerevisiae. Mol Cell Biol 16: 2483-2495.

Lynch PI, Rusche LN. 2009. A silencer promotes the assembly of silenced chromatin independently of recruitment. Mol Cell Biol 29: 43-56.

Martino F, Kueng S, Robinson P, Tsai-Pflugfelder M, van Leeuwen F, Ziegler M, Cubizolles F, Cockell MM, Rhodes D, Gasser SM. 2009. Reconstitution of yeast silent chromatin: Multiple contact sites and O-AADPR binding load SIR complexes onto nucleosomes in vitro. Mol Cell 33: 323-334.

McBryant SJ, Krause C, Hansen JC. 2006. Domain organization and quaternary structure of the Saccharomyces cerevisiae silent information regulator 3 protein, Sir3p. Biochemistry 45: 15941-15948.

Mondoux MA, Zakian VA. 2007. Subtelomeric elements influence but do not determine silencing levels at Saccharomyces cerevisiae telomeres. Genetics 177: 2541-2546.

Neuwald AF, Aravind L, Spouge JL, Koonin EV. 1999. AAA ${ }^{+}$: A class of chaperone-like ATPases associated with the assembly, operation, and disassembly of protein complexes. Genome Res 9: 27-43.

Norris A, Bianchet MA, Boeke JD. 2008. Compensatory interactions between Sir3p and the nucleosomal LRS surface imply their direct interaction. PLoS Genet 4: e1000301. doi: 10.1371/journal.pgen.1000301.

Ohno S. 1970. Evolution by gene duplication. Springer, Berlin.

Onishi M, Liou GG, Buchberger JR, Walz T, Moazed D. 2007. Role of the conserved Sir3-BAH domain in nucleosome binding and silent chromatin assembly. Mol Cell 28: 10151028.

Park Y, Hanish J, Lustig AJ. 1998. Sir3p domains involved in the initiation of telomeric silencing in Saccharomyces cerevisiae. Genetics 150: 977-986.

Pryde FE, Louis EJ. 1999. Limitations of silencing at native yeast telomeres. EMBO J 18: 2538-2550.

Rudner AD, Hall BE, Ellenberger T, Moazed D. 2005. A nonhistone protein-protein interaction required for assembly of the SIR complex and silent chromatin. Mol Cell Biol 25: 4514-4528.

Rusche LN, Kirchmaier AL, Rine J. 2002. Ordered nucleation and spreading of silenced chromatin in Saccharomyces cerevisiae. Mol Biol Cell 13: 2207-2222.

Rusche LN, Kirchmaier AL, Rine J. 2003. The establishment, inheritance, and function of silenced chromatin in Saccharomyces cerevisiae. Annu Rev Biochem 72: 481-516.

Sampath V, Yuan P, Wang IX, Prugar E, van Leeuwen F, Sternglanz R. 2009. Mutational analysis of the Sir3 BAH domain reveals multiple points of interaction with nucleosomes. Mol Cell Biol 29: 2532-2545.

Sauve AA, Celic I, Avalos J, Deng H, Boeke JD, Schramm VL. 2001. Chemistry of gene silencing: The mechanism of $\mathrm{NAD}^{+}$-dependent deacetylation reactions. Biochemistry 40: 15456-15463.

Southey, R. 1834. The doctor. Harper, NY.

Stone EM, Reifsnyder C, McVey M, Gazo B, Pillus L. 2000. Two classes of sir3 mutants enhance the sirl mutant mating defect and abolish telomeric silencing in Saccharomyces cerevisiae. Genetics 155: 509-522.

Strahl-Bolsinger S, Hecht A, Luo K, Grunstein M. 1997. SIR2 and SIR4 interactions differ in core and extended telomeric heterochromatin in yeast. Genes \& Dev 11: 83-93.

Tanner KG, Landry J, Sternglanz R, Denu JM. 2000. Silent information regulator 2 family of NAD-dependent histone/ protein deacetylases generates a unique product, 1-O-acetylADP-ribose. Proc Natl Acad Sci 97: 14178-14182.
Tanny JC, Kirkpatrick DS, Gerber SA, Gygi SP, Moazed D. 2004. Budding yeast silencing complexes and regulation of Sir2 activity by protein-protein interactions. Mol Cell Biol 24: 6931-6946.

Thompson JS, Snow ML, Giles S, McPherson LE, Grunstein M. 2003. Identification of a functional domain within the essential core of histone $\mathrm{H} 3$ that is required for telomeric and HM silencing in Saccharomyces cerevisiae. Genetics 163: 447-452.

van Leeuwen F, Gottschling DE. 2002. Genome-wide histone modifications: Gaining specificity by preventing promiscuity. Curr Opin Cell Biol 14: 756-762.

van Leeuwen F, Gafken PR, Gottschling DE. 2002. Dotlp modulates silencing in yeast by methylation of the nucleosome core. Cell 109: 745-756.

van Welsem T, Frederiks F, Verzijlbergen KF, Faber AW, Nelson ZW, Egan DA, Gottschling DE, van Leeuwen F. 2008. Synthetic lethal screens identify gene silencing processes in yeast and implicate the acetylated amino terminus of Sir3 in recognition of the nucleosome core. Mol Cell Biol 28: 38613872 .

Venkatasubrahmanyam S, Hwang WW, Meneghini MD, Tong AH, Madhani HD. 2007. Genome-wide, as opposed to local, antisilencing is mediated redundantly by the euchromatic factors Set1 and H2A.Z. Proc Natl Acad Sci 104: 1660916614.

Wang X, Connelly JJ, Wang CL, Sternglanz R. 2004. Importance of the Sir3 $\mathrm{N}$ terminus and its acetylation for yeast transcriptional silencing. Genetics 168: 547-551.

Xu F, Zhang Q, Zhang K, Xie W, Grunstein M. 2007. Sir2 deacetylates histone $\mathrm{H} 3$ lysine 56 to regulate telomeric heterochromatin structure in yeast. Mol Cell 27: 890-900. 


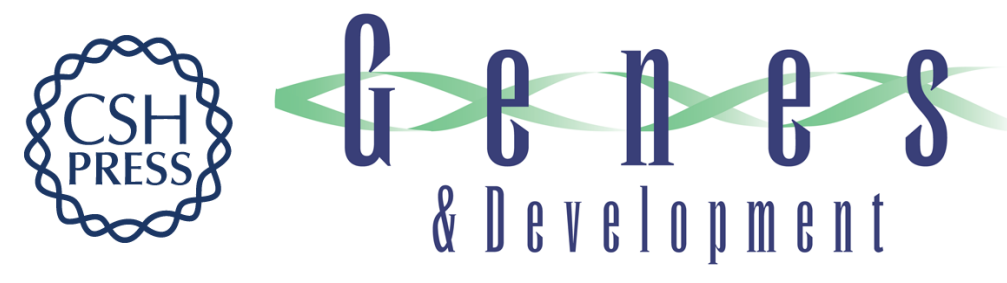

\section{Silent information regulator 3: the Goldilocks of the silencing complex}

Anne Norris and Jef D. Boeke

Genes Dev. 2010, 24:

Access the most recent version at doi:10.1101/gad.1865510

Supplemental
Material http://genesdev.cshlp.org/content/suppl/2009/12/30/24.2.115.DC1

References This article cites 54 articles, 25 of which can be accessed free at: http://genesdev.cshlp.org/content/24/2/115.full.html\#ref-list-1

License

Email Alerting Receive free email alerts when new articles cite this article - sign up in the box at the top Service right corner of the article or click here.

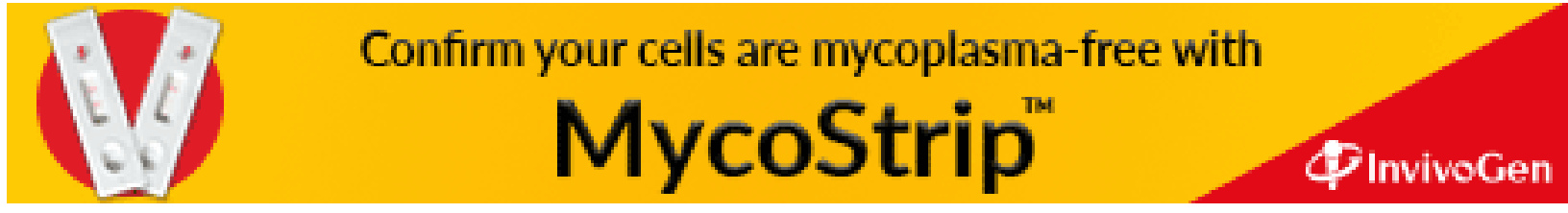

